\title{
Influence of the projectile field on free target electrons
}

\author{
D. H. Jakubaßa-Amundsen \\ Physics Section, University of Munich, 85748 Garching, Germany
}

(Received 30 June 1993)

\begin{abstract}
The spectral distribution of loosely bound target electrons emitted in collisions with fast bare projectiles is described in terms of electron capture to the projectile continuum. Comparison with experimental data on electron ejection from $\mathrm{He}$ by $1-5-\mathrm{MeV} / \mathrm{amu} \mathrm{H}^{+}, \mathrm{C}^{6+}, \mathrm{O}^{8+}$, and $\mathrm{F}^{9+}$ impact into forward directions shows that the impulse approximation for rearrangement is able to describe the shape of the energy distribution not only at the cusp, but also in the binary-encounter region and beyond. The results of the post form of the impulse approximation are discussed in relation to the first-order Born approximation, the continuum distorted-wave theory, the fully peaked prior impulse approximation, and in particular the recently presented distorted-wave strong-potential Born approximation.
\end{abstract}

PACS number(s): 34.50.Fa, 34.70. $+\mathrm{e}$

\section{INTRODUCTION}

The spectroscopy of electrons emitted in energetic ion-atom collisions is a powerful tool to gain insight into the collision dynamics and into the electronic structure of the collision partners. Apart from the high intensity of low-energy electrons, the energy distribution of ejected target electrons shows two features, the forward peak or "cusp" at an energy $E_{f}=v^{2} / 2$ for emission angles $\vartheta_{f}$ close to zero degrees, and the binary-encounter peak near $E_{f}^{*}=2 v^{2} \cos ^{2} \vartheta_{f}+E_{i}$ where $v$ denotes the collision velocity and $E_{i}$ the bound-state energy of the electron under consideration. These structures in the doubly differential cross section for electron ejection provide a sensitive test of the theoretical models. While it had been realized long ago that the forward peak requires a higher-order theory which accounts for the final-state interaction with the projectile [1,2], the binary-encounter peak is conventionally described by the first-order Born approximation for direct ionization [3].

Stimulated by recent experimental investigations $[4,5]$ of the binary-encounter peak which revealed deficiencies of the Born approximation concerning the precise peak position, theories have been put forth which consider the influence of the projectile field on the binary-encounter electrons and hence allow for a unified description of both features in the electron spectra. Two types of theories may be distinguished which include the interaction between the electron and the bare projectile nonperturbatively in the electronic final state. To the first type belong the distorted-wave theories which represent the final electronic state by a product of eigenfunctions to projectile and target like the continuum distorted-wave (CDW) approximation [6,7] or the distorted-wave impulse approximation [8]. What these theories have in common is that they consider the final-state interaction with both projectile and target field nonperturbatively, which implies that they not only can account for the for- ward peak, but also that they are able to reproduce the intensity of the low-energy electrons. However, the deficiency of this type of theories which satisfy the correct asymptotic Coulomb boundary conditions at the expense of being not quite accurate in the interaction zone becomes evident when one of the nuclear fields is strongly dominating. For example, the CDW theory does not correctly reproduce the cusp asymmetry.

The second type of theories neglects the target influence on the electronic final state completely. To these belong the first-order (Brinkman-Kramers) theory for rearrangement which, however, not only fails to describe the cusp asymmetry but also overestimates systematically the binary-encounter peak intensity [9]. More advanced theories are the impulse approximation (IA) [10-12] where the electron transfer proceeds via an intermediate projectile eigenstate (post IA) or target eigenstate (prior IA), and the distorted-wave strongpotential Born (DSPB) theory [13] which accounts for the off-shell propagation of the electron in its intermediate state. These theories are able to describe the cusp asymmetry and the binary-encounter peak intensity $[9,11]$, but fail to reproduce the low-energy part of the electron spectra. This is so because the low-energy electrons are dominated by the target field, while the binary-encounter electrons are basically field-free such that their intensity can rather well be described by the plane-wave Born or binary-encounter approximation [4]. It should be noted, however, that full peaking approximations may change these properties of the IA or DSPB. For example, the fully peaked prior IA rather belongs to the first type of theories (it has originally been derived from a highenergy CDW formulation [1]), and hence is fairly good in describing the low-energy electrons.

In the present work, the post impulse approximation which up to now has mostly been applied to cusp electrons, is used for the interpretation of the high-energy part of the electron spectra $\left(E_{f}>0.1 \mathrm{keV}\right)$ for forward emission angles up to $60^{\circ}$. In Sec. II, this theory is com- 
pared with the DSPB approach with particular emphasis on the peaking approximations and wave-function prescriptions used in the actual calculations $[9,11]$. In Sec. III, the post IA is tested against the experimental data [4,14-18] and compared with first-order Born, fully peaked prior IA and available CDW results [19-21]. A summary is given in Sec. IV. Atomic units $(\hbar=m=e=1)$ are used unless otherwise indicated.

\section{THE POST IMPULSE APPROXIMATION AND THE DSPB APPROACH}

The impulse approximation is a theory well suited for the description of electron transfer in fast collisions between asymmetric collision partners with $Z_{P}<<Z_{T}$ or $Z_{P} \gg Z_{T}$ where $Z_{P}$ and $Z_{T}$ are the nuclear charges of the projectile and the target. It is a first-order perturbative theory in the weaker of the two potentials, $V_{P}$ and $V_{T}$, which act on the electron. Consequently, the IA exists in two nonequivalent forms, the prior form for $Z_{P} \lesssim Z_{T}$ and the post form for $Z_{P} \gtrsim Z_{T}$, which at asymptotically high collision velocities $v$ both coincide with the second-order Born theory for rearrangement. For heavy projectiles $\left(Z_{P} \gtrsim Z_{T}\right)$, electron transfer to a projectile continuum state is described in the following way. The active electron, being dressed with the projectile field and having a momentum $\mathbf{q}^{\prime}$, scatters inelastically on the target core potential $V_{T}$. The distribution of $\mathrm{q}^{\prime}$ is obtained from the initially bound target state $\psi_{i}^{T}$ in momentum space. In the semiclassical independent-particle approximation, the transition amplitude reads

$$
a_{f i}^{\text {post IA }}=-i \int_{-\infty}^{\infty} d t \int d \mathbf{q}^{\prime}\left\langle\psi_{f}^{P}\left|V_{T}\right| \psi_{\mathbf{q}^{\prime}}^{P}\right\rangle\left\langle\mathbf{q}^{\prime} \mid \psi_{i}^{T}\right\rangle,
$$

where $\left|\mathbf{q}^{\prime}\right\rangle$ is a plane wave of momentum $\mathbf{q}^{\prime}$ in the projectile frame of reference, and $\psi_{\mathbf{q}^{\prime}}^{P}$ and $\psi_{f}^{P}$ are continuum projectile eigenstates with momenta $\mathbf{q}^{\prime}$ and $\boldsymbol{\kappa}_{f}=\mathbf{k}_{f}-\mathbf{v}$, respectively ( $\mathbf{k}_{f}$ is the electron's final momentum in the target frame of reference). Using a straight-line path for the internuclear motion, the doubly differential cross section can be written in the following way [11]:

$$
\frac{d^{2} \sigma^{\text {post IA }}}{d E_{f} d \Omega_{f}}=\frac{2 \pi k_{f}}{v} \int d \mathbf{q} \delta\left(\mathbf{q v}+k_{f}^{2} / 2-E_{i}\right)\left|\int d \mathbf{s} \varphi_{i}^{T}(\mathbf{s}+\mathbf{v}) \widetilde{V}_{T}\left(\mathbf{q}+\mathbf{k}_{f}-\mathbf{v}-\mathbf{s}\right) M(\mathbf{q}, \mathbf{s})\right|^{2}
$$

where we have introduced the Fourier transform $\widetilde{V}_{T}$ of the target core potential, the collision velocity $\mathbf{v}$, and the initial-state energy $E_{i}$. The scattering matrix element is given by

$$
\boldsymbol{M}(\mathbf{q}, \mathbf{s})=\left\langle\psi \boldsymbol{\kappa}_{f}^{P}(\mathbf{r})\left|e^{i\left(\mathbf{q}+\mathbf{k}_{f}-\mathbf{v}-\mathbf{s}\right) \mathbf{r}}\right| \psi_{\mathbf{s}}^{P}(\mathbf{r})\right\rangle,
$$

where for a bare projectile, the intermediate state $\psi_{\mathrm{s}}^{P}$ is an incoming Coulomb wave

$$
\begin{aligned}
& \psi_{\mathbf{s}}^{P}(\mathbf{r})=(2 \pi)^{-3 / 2} N\left(\eta_{s}\right) e_{1}^{i \mathbf{s} \cdot \mathbf{r}} F_{1}\left(i \eta_{s}, 1, i(s r-\mathbf{s} \cdot \mathbf{r})\right), \\
& N\left(\eta_{\mathbf{s}}\right)=e^{\pi \eta_{s} / 2} \Gamma\left(1-i \eta_{s}\right),
\end{aligned}
$$

with $\eta_{s}=Z_{P} / s$ and ${ }_{1} F_{1}$ a confluent hypergeometric function.

For the evaluation of (2.2), a peaking approximation can be introduced since for light targets, the bound-state momentum distribution $\varphi_{i}^{T}(\mathbf{s}+\mathbf{v})$ is strongly peaked at $\mathbf{s}=-\mathbf{v}$. The so-called full peaking approximation which would consist in replacing $s$ by $-v$ in $M(q, s)$ can, however, not be used because it would make the cross section diverge. A more sophisticated approximation is the socalled transverse peaking approximation which only affects the components of $s$ perpendicular to $v$. This peaking is applied to $\psi_{\mathbf{s}}^{P}(\mathbf{r})$ and consists in the replacement $\mathbf{s} \rightarrow s_{z} \mathbf{e}_{z}$ ( $\mathbf{e}_{z}$ being a unit vector in beam direction) in $\eta_{s}$ as well as in the argument $s r-\mathbf{s} \cdot \mathbf{r}$ of the function ${ }_{1} F_{1}$. Since the phase factor $\exp (i \mathbf{S} \cdot \mathbf{r})$ is canceled by the transition operator in (2.3), the transition matrix element $\boldsymbol{M}(\mathbf{q}, \mathbf{s})$ will then only depend on $s_{z}$, while the remaining integral in (2.2) over the transverse components $s_{\perp}$ can be done analytically [11]. This transverse peaking does not restrict the validity region of the post IA.
The distorted-wave strong potential Born theory is also a first-order perturbative theory in the weak target field, but in contrast to the IA, it includes distortion potentials in the initial and final channels and treats the intermediate electronic state off-shell [13]. An exact evaluation of the DSPB is even more involved than is the case for the IA. Hence the approximations made in the numerical calculations [9] concern the neglect of off-shell effects and distortion potentials after the elimination of the singularity in the elastic channel [13], such that the DSPB in fact reduces to the post IA, Eqs. (2.2)-(2.4). In their best approach, termed "DSPB without peaking," Brauner and Macek [9] apply two further approximations: In the intermediate state (2.4), they make the full peaking $s=v$ in $\eta_{s}$, and the transverse peaking $s=-\mathbf{s e}_{z}=\left|s_{z}\right|$ in part of the argument of the hypergeometric function. Hence, the actual DSPB results are obtained from (2.2) and (2.3) with the approximate function

$$
\psi_{\mathbf{s}}^{P}(\mathbf{r}) \approx(2 \pi)^{-3 / 2} N\left(\eta_{v}\right) e_{1}^{i \mathbf{s} \cdot \mathbf{r}} F_{1}\left(i \eta_{v}, 1, i\left(\left|s_{z}\right| r-\mathbf{s} \cdot \mathbf{r}\right)\right),
$$

where $\eta_{v}=Z_{P} / v$. As compared to the transverse peaked IA which has numerical instabilities in the cusp region due to a strong singularity in the integrand (we had to extrapolate the cross sections at zero degree from those at larger angles), the above DSPB approach allows for an analytic evaluation of the $s$ integral (at the expense of a one-dimensional auxiliary integral) and hence facilitates the calculations considerably.

In Fig. 1 results from the transverse peaked post IA and the DSPB approach are compared with each other for $1.5-\mathrm{MeV} / \mathrm{amu} \mathrm{H}^{+}$and $\mathrm{F}^{9+}$ on $\mathrm{He}$ at an emission an- 
gle of zero degree. In order to exclude wave function effects in the comparison of the two models, the BatesGriffing prescription [3] which had been chosen for the DSPB is also taken for the IA, implying the use of a hydrogenic target wave function as well as a hydrogenic target core potential with an effective charge $Z_{T \text {, eff }}$ $=\left(2\left|E_{i}\right|\right)^{1 / 2}=1.345$. In fact, due to the different type of peaking approximations, the two theories differ considerably, both in predicting the electron yield (except in the region of the binary-encounter peak at $3.15 \mathrm{keV}$ ) and the cusp asymmetry. This behavior can readily be understood from investigating the validity of the full peaking approximation of the DSPB in the principal region of the $s$ integrand in (2.2). The binary encounter peak, for example, is obtained by setting $\mathbf{s}+\mathbf{v}=0$ in the bound-state function $\varphi_{i}^{T}(\mathbf{s}+\mathbf{v})$ together with $\mathbf{q}=-\mathbf{k}_{f}$ where (for $\mathbf{s}=-\mathbf{v})$ the target potential $\widetilde{V}_{T}\left(\mathbf{q}+\mathbf{k}_{f}-\mathbf{v}-\mathbf{s}\right)$ is peaked. From the energy-conserving $\delta$ function one then obtains the approximate binary-encounter energy $\mathbf{k}_{f}^{2} / 2=E_{f}^{*}=2 v^{2} \cos ^{2} \vartheta_{f}+E_{i}$. Also the matrix element $M(\mathbf{q}, \mathbf{s})$ is peaked for this choice of momenta [11]. Hence, the full peaking approximation is very good in the vicinity of the binary-encounter peak, and the two theories give similar results.

The situation is different for electrons at lower energy. Let us select the cusp region with $\mathbf{k}_{f}=\mathbf{v}$. From the $\delta$ function it follows that $\mathrm{q} \approx-\mathrm{v} / 2$ (for $\left|E_{i}\right|$ small) and hence $\mathbf{s} \approx-\mathbf{v} / 2$ from the maximum of the target potential. Additional consideration of the peaking condition [11] of the matrix element, $\mathbf{s} \cdot \mathbf{q}=q^{2} / 2$, will lead to a fur- ther reduction of $s$. Hence, the full peaking approximation is rather poor. Taken into consideration that the normalization factor $N\left(\eta_{s}\right)$ of the Coulomb wave $\psi_{\mathrm{s}}^{P}$ decreases in magnitude when $s$ is increased, the full peaking (i.e., DSPB) results are expected to be below the transverse peaked IA results (which permit a variation of $\mathbf{s}$ in the direction of $\mathbf{v}$ ), the more, the larger $Z_{P}$. This is confirmed in the calculations of Fig. 1, and also by artificially restricting the $s_{z}$ integration in the transverse peaked IA to the interval $(-2 v,-0.7 v)$ instead of using the interval $(-2 v,-0.25 v)$ which is sufficient for good convergence. For both projectiles, this restriction changes the binary encounter peak intensity by less than $5 \%$, but leads to a reduction of $20-45 \%$ for $E_{f} \lesssim 2.3$ $\mathrm{keV}$. Of course, the deficiencies of the full peaking approximation are the less severe, the higher the impact energy.

Having investigated the effects of the peaking approximation we now turn to the sensitivity of the numerical results to the choice of wave functions and potentials. Rather than using the Bates-Griffing hydrogenic model, one should employ the Hartree-Fock prescription for the two-electron helium target. Transverse-peaked post IA calculations with a Clementi-Roetti bound-state function, an effective screened helium core potential [11], and the experimental binding energy $\left(E_{i}=-0.918\right.$ a.u. $)$ are included in Fig. 1. It is readily seen that the so obtained cross sections (which are in good agreement with experiment except below the cusp where the capture to continuum prescription begins to fail, cf. Fig. 2) are in general

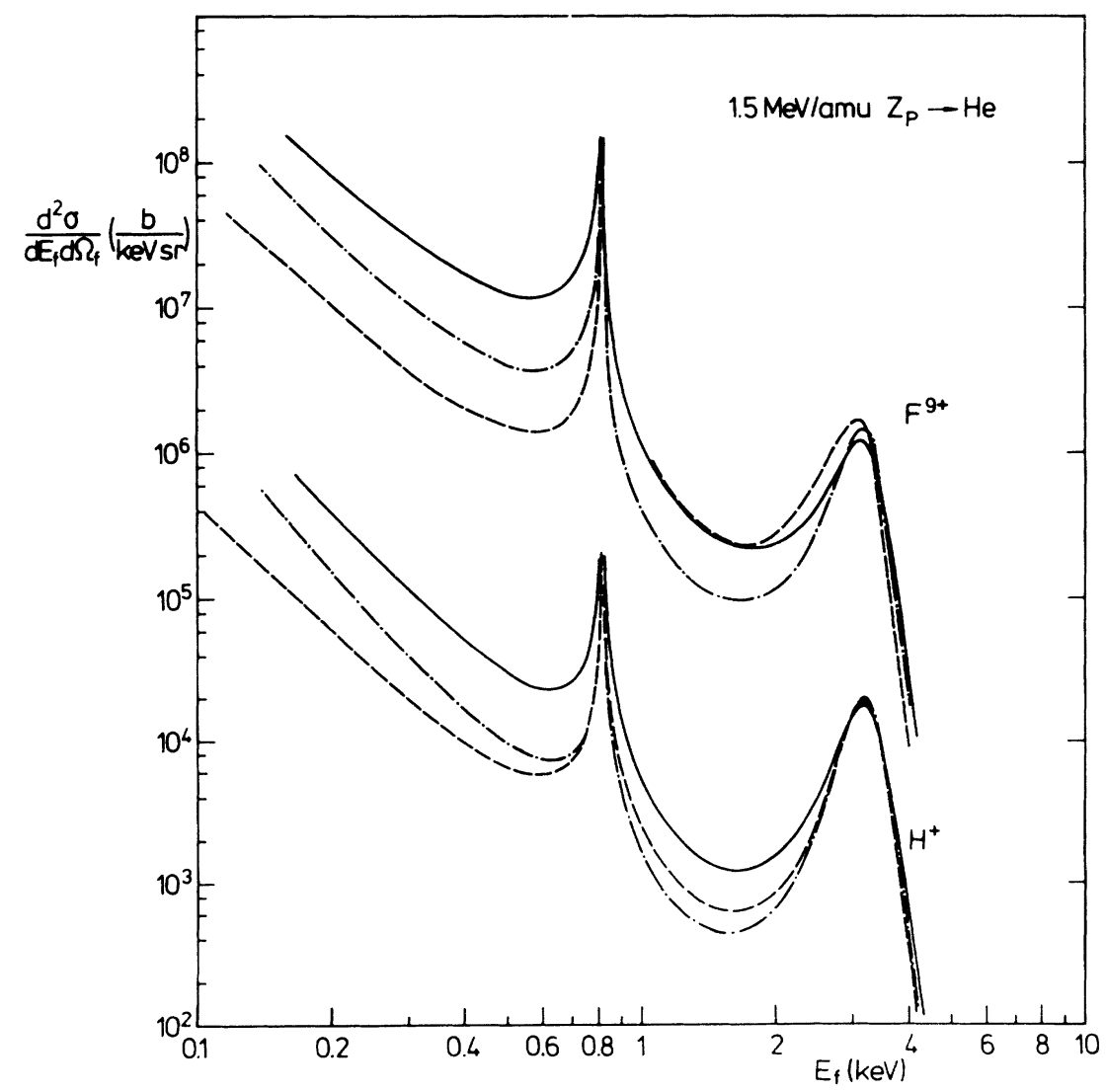

FIG. 1. Doubly differential cross section for electron emission in $1.5 \mathrm{MeV} / \mathrm{amu} \mathrm{F}^{9+}+\mathrm{He}$ and $\mathrm{H}^{+}+\mathrm{He}$ collisions at forward direction $\left(\vartheta_{f}=0^{\circ}\right)$ as a function of electron emission energy $E_{f}$. pulse approximation according to Ref. [11]. - - , DSPB results from Fig. 5 of Ref. [9]. $-\cdot-\cdot-\cdot$, transverse peaked post IA with hydrogenic target wave function and potential. 


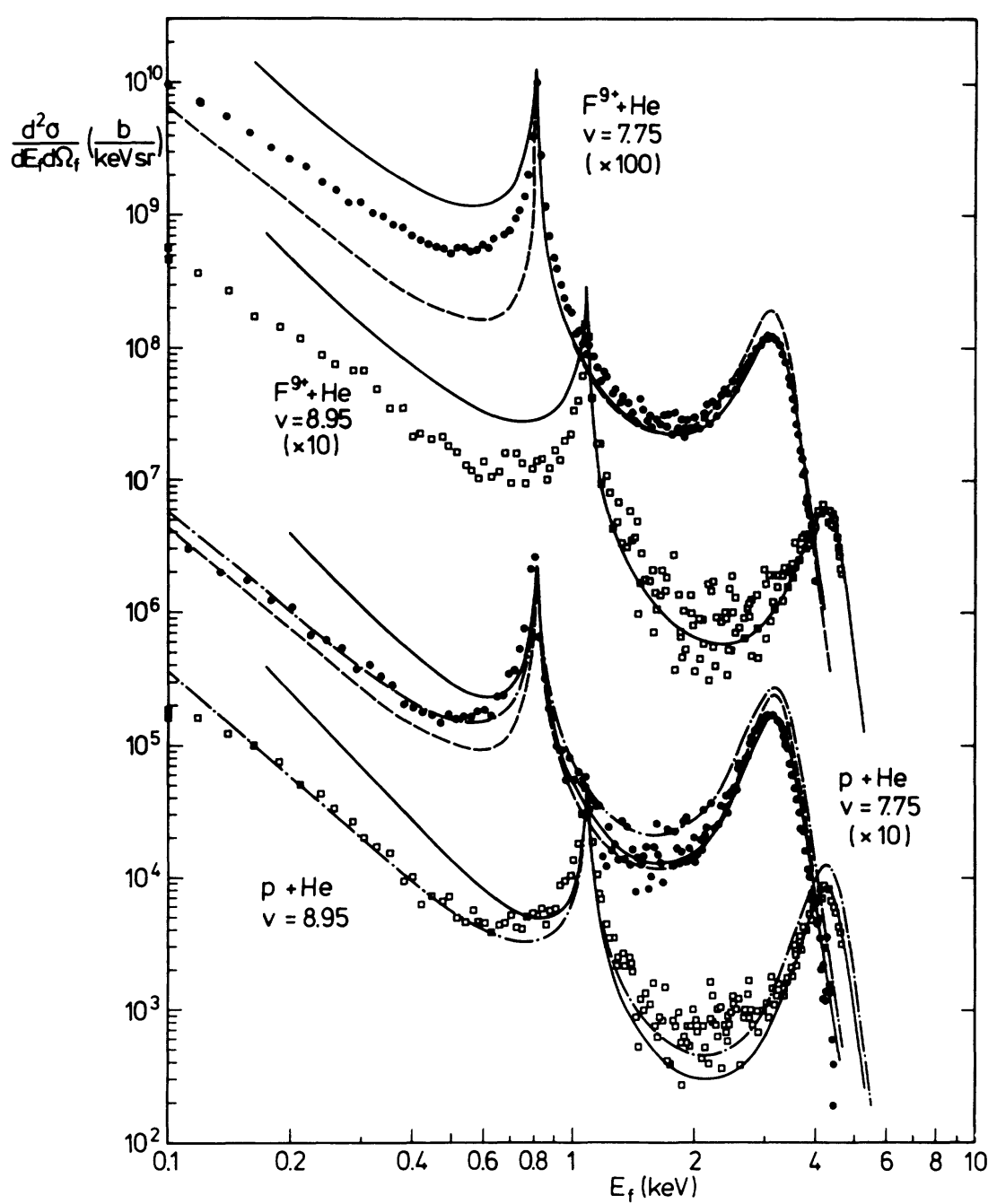

FIG. 2. Doubly differential cross section for $1.5 \mathrm{MeV} / \mathrm{amu}(v=7.75$ a.u.) and $2 \mathrm{MeV} / \mathrm{amu}$ $(v=8.95$ a.u. $) \mathrm{F}^{9+}+\mathrm{He}$ and $\mathrm{H}^{+}+\mathrm{He}$ collisions at forward direction $\left(\vartheta_{f}=0^{\circ}\right)$. Experiment: 0 , Lee et al. [4], $\square$ Lee and Richard [14]. Theory: — transverse peaked post IA, $-\cdot-\cdot-\cdot$ fully peaked prior IA, using an angular acceptance of $\theta_{0}=0.6^{\circ}$ (which is an average of the strongly nonisotropic experimental angular acceptance). - - CDW-EIS calculation of Fainstein, Ponce, and Rivarola [19] (without average over $\theta_{0}$ ). much larger than the hydrogenic results. In order to separate the influence of an improved wave function from the influence of the screening in the potential, we have tentatively used a hydrogenlike wave function but a screened helium core potential for $\mathrm{H}^{+}+\mathrm{He}$. The corresponding results lie for most electron energies about halfway between the hydrogenic and the nonhydrogenic IA cross sections (except at the binary encounter peak where they are about $10 \%$ below the nonhydrogenic results), demonstrating that a proper choice of wave function and potential is equally important. The large difference between the results from an improved target prescription and the hydrogenic case persists when the collision velocity is increased, such that an influence from possible inaccuracies of the transverse peaking approximation can be excluded. Rather, as has been pointed out by Madsen and Taulbjerg [22], electron emission in the region well below the binary-encounter peak is governed by the extreme wings of the target Compton profile. These wings are very sensitive to the prescription used for the target atom, and so are the corresponding electron intensities.

\section{THE POST IA IN COMPARISON WITH OTHER THEORIES AND WITH EXPERIMENT}

We have calculated electron spectra for $0.5,1,1.5,2$, and 4.2-MeV $\mathrm{H}^{+}+\mathrm{He}$ at emission angles between $0^{\circ}$ and $30^{\circ}$, for $1.5-\mathrm{MeV} / \mathrm{amu}$ and $2-\mathrm{MeV} / \mathrm{amu} \mathrm{F}^{9+}+\mathrm{He}$ at zero degree and for 5-MeV/amu $\mathrm{C}^{6+}$ and $\mathrm{O}^{8+}$ on $\mathrm{He}$ at $50^{\circ}$ and $60^{\circ}$ where experimental data are available which extend beyond the binary-encounter region [4,14-18]. In the cusp region, the zero-degree spectra are averaged over the angular acceptance $\theta_{0}$ of the detector. Throughout, the post form of the impulse approximation is evaluated with the help of the transverse peaking approximation, using the Hartree-Fock target prescription from Ref. [11]. Figure 2 compares the calculated zerodegree electron spectra from 1.5-MeV/amu and 2$\mathrm{MeV} / \mathrm{amu} \mathrm{F}^{9+}$ and $\mathrm{H}^{+}$impact on $\mathrm{He}$ with the experimental data from Lee and co-workers $[4,14]$. These data have not been measured on an absolute scale and hence are normalized to the post IA cross sections in the binary-encounter peak maximum. We will argue below that for proton impact, the data should lie about $15-20 \%$ higher, which is a similar value as given in the original work [4]. Even for proton impact, the post IA gives a good description of the data above the cusp (which for the two collision velocities is located at 0.871 and $1.09 \mathrm{keV}$, respectively). At the smaller energies where the influence of the target field on the ejected electron gains increasing importance, the post IA overestimates experiment considerably. The lack of inclusion of this final-state interaction also leads to an overprediction 
of the cusp asymmetry for the heavy $\mathrm{F}^{9+}$ projectiles at these rather low velocities.

For the weakly asymmetric $\mathrm{H}^{+}+\mathrm{He}$ system, the prior impulse approximation should conceptually be more appropriate. In this theory, suited for light projectiles $\left(Z_{p} \lesssim Z_{T}\right)$, electron transfer is described in terms of direct ionization from the initial state $\psi_{i}^{T}$ to an intermediate continuum target eigenstate $\psi_{q}^{T}$ with a momentum q close to the electronic momentum $\mathbf{k}_{f}$, followed by an overlap with the final projectile eigenstate $\psi_{f}^{P}$,

$a_{f i}^{\text {prior IA }}=-i \int_{-\infty}^{\infty} d t \int d \mathbf{q}\left\langle\psi_{f}^{P} \mid \mathbf{q}\right\rangle\left\langle\psi_{\mathbf{q}}^{T}\left|V_{P}\right| \psi_{i}^{T}\right\rangle$,

where $V_{P}$ is the interaction with the projectile nucleus and $|\mathbf{q}\rangle$ is a plane wave of momentum $\mathbf{q}$ in the target frame of reference. However, it has been argued by Macek [23] that in the binary-encounter peak region, the prior IA is likely to be inferior to the post IA because the latter can be shown [24] to reduce to the plane-wave Born formula (which describes the peak in terms of the Rutherford cross section times the initial-state Compton profile), while the prior IA does not. In fact, applying the full peaking approximation to the ionization matrix element leads to the following factorization of the differential cross section [12] $\frac{d^{2} \sigma^{\text {prior IA }}}{d E_{f} d \Omega_{f}} \approx\left|N\left(\eta_{f}\right)\right|^{2} \frac{d^{2} \sigma^{B 1}}{d E_{f} d \Omega_{f}}, \quad \eta_{f}=Z_{P} / \kappa_{f}$,

where $d^{2} \sigma^{B 1} / d E_{f} d \Omega_{f}$ is the first-order Born cross section for direct ionization, which is multiplied by the Coulomb normalization factor defined in (2.4). For intermediate velocities this factor, which measures the deviation from the plane-wave Born result, may be quite large. However, in the unpeaked version of the IA, this factor is attenuated through the folding of the final-state momentum space wave function with the ionization matrix element, such that the exact prior IA will be closer to the Born result.

Since the fully peaked prior IA has been frequently applied to electron emission induced by light projectiles $[12,25]$, we have included this theory in our present study. For the prior IA (as well as for the first-order Born calculations) Slater-screened hydrogenic boundstate and continuum target functions (with $Z_{T, \text { eff }}=1.7$ ) have been used which fulfill the requirement of orthogonality that is necessary for a correct evaluation of the ionization matrix element. It is seen from Fig. 2 that even at $2-\mathrm{MeV}$ proton impact, one is not yet in the validity regime of the full peaking approximation, and hence

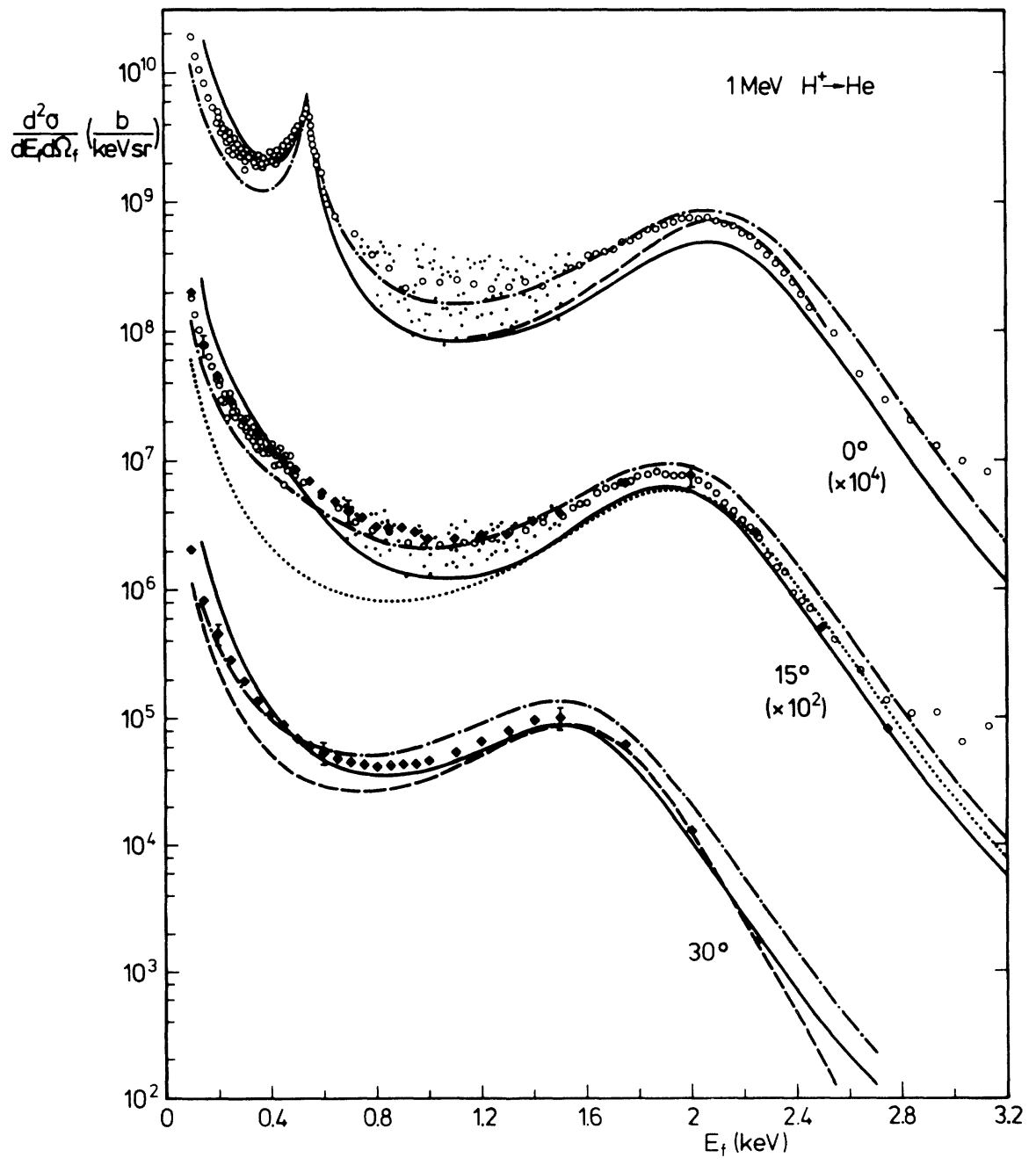

FIG. 3. Doubly differential cross section for electron emission in $1-\mathrm{MeV} \mathrm{H}^{+}+\mathrm{He}$ collisions at emission angles $0^{\circ}, 15^{\circ}$, and $30^{\circ}$. Experiment: $\bigcirc$ Fiedler, Kuzel, and Groeneveld [18], Rudd, Toburen, and Stolterfoht [17], data averaged over a certain energy range; - Fiedler, Kuzel, and Groeneveld [18], selection of nonaveraged data points. Theory: — transverse peaked post IA, -..... fully peaked prior IA, - CDW-EIS from Fainstein, Ponce, and Rivarola [20,21], . . . first-order Born. For the $0^{\circ}$ data, $\theta_{0}=1.67^{\circ}$. 
our prior IA results overestimate experiment at the binary-encounter peak by $30-40 \%$ and they also do not predict the correct cusp asymmetry. Below the cusp, on the other hand, there is quite good agreement between the fully peaked prior IA and experiment. This can be attributed to the fact that the full peaking approximation implies a change of the boundary conditions since the intermediate target eigenstate has fully acquired the properties of a final target state. That is necessary for an adequate description of the low-energy electron emission. On the other hand, the unpeaked prior IA as a theory for charge transfer to the projectile, would not provide the correct behavior at $E_{f} \rightarrow 0$. This discrepancy between the fully peaked and the unpeaked prior IA persists even at ultrahigh collision velocities. Shown in Fig. 2 are also results of the CDW-EIS (eikonal initial state) theory of Fainstein, Ponce, and Rivarola [19]. Like the fully peaked prior IA, this theory overestimates the binaryencounter peak intensity and predicts only a weakly asymmetric cusp, whereas (at least for proton impact) the low-energy region is rather well reproduced.

In order to check the data normalization to the post IA and to study the behavior at larger emission angles, we show in Figs. 3 and 4 absolutely measured $[17,18]$ electron spectra for $1 \mathrm{MeV}$ and $1.5-\mathrm{MeV} \mathrm{H}^{+}+\mathrm{He}$ in the angular range from $0^{\circ}$ to $30^{\circ}$, in comparison with theory.
From consideration of the binary-encounter peak for 1$\mathrm{MeV}$ proton impact it follows that the post IA underestimates experiment by $35 \%, 20 \%$, and $10 \%$ for the angles $0^{\circ}, 15^{\circ}$, and $30^{\circ}$, respectively. At $1.5 \mathrm{MeV}$ the agreement is somewhat improved, the deviations being $15 \%$ at $15^{\circ}$ and $30^{\circ}$. From this we conjecture that in Fig. 2, the 1.5$\mathrm{MeV} p+\mathrm{He}$ data should presumably lie between 15-25\% higher than shown, and the 2-MeV $p+$ He data about $10-15 \%$. However, one should keep in mind that the experimental cross sections in Figs. 3 and 4 have an absolute uncertainty of $20 \%$ (the data from Ref. 18 are normalized to the Rudd, Toburen, and Stolterfoht [17] data).

When the emission angle is increased (but the electron energy kept fixed), the influence of the target field on the emitted electron gets stronger. Consequently, the firstorder Born approximation is in better accord with the data. For the smaller emission angles, this is in general also true for the post IA, which is particularly close to the Born approximation near the binary-encounter peak. This agreement in the binary-encounter peak intensity is expected from the properties of the post IA [24]. (However, when the emission angle is further increased into the backward hemisphere where the target field is still more important, the post IA will give poorer results.) As far as the valley between the low-energy electrons and the

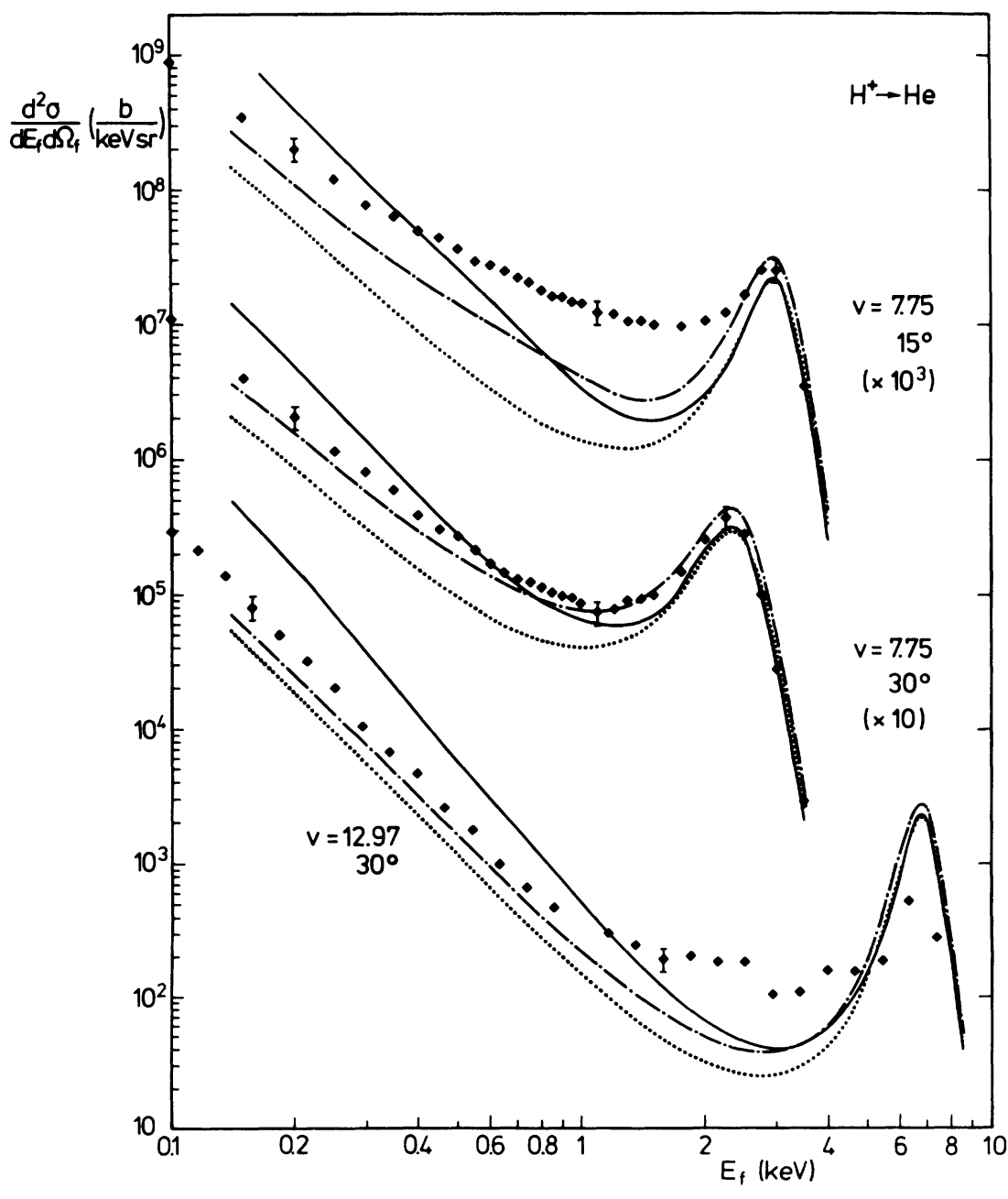

FIG. 4. Doubly differential cross section for electron emission in $1.5-\mathrm{MeV} \mathrm{H}^{+}+\mathrm{He}$ collisions at $\vartheta_{f}=15^{\circ}$ and $30^{\circ}$, and in $4.2-\mathrm{MeV}$ $(v=12.97$ a.u. $) \mathrm{H}^{+}+\mathrm{He}$ collisions at $\vartheta_{f}=30^{\circ}$. Experiment and theory, see caption of Fig. 3. 
binary-encounter electrons is concerned, there does not seem to be any systematically improved description of the data by the impulse approximation when the angle or the collision velocity is increased. On the other hand, as indicated in Fig. 3, the data show a large spread in this region which is the larger, the deeper the valley (i.e., the smaller $\vartheta_{f}$ or the higher $v$ ). Hence, there may be ambiguities when comparing with data which are averaged over a certain energy interval like those of Rudd, Toburen, and Stolterfoht [17].

If comparison between theory and experiment is restricted to the binary-encounter region, there is a distinct trend with the collision energy. When it is decreased below $1.5 \mathrm{MeV}$, the post IA underestimates the peak intensity, the more, the lower $v$. This indicates the breakdown of the transverse peaking approximation, as well as of the post IA itself. For such low collision energies, the fully peaked prior IA gives cross sections well above the post IA ones. Due to the consideration of two-center final-state effects, this theory is in better agreement with the data. In order to show that the post-prior discrepancy decreases when the collision gets more energetic (except for the low-energy electrons) we have included in Fig. 4 an electron spectrum for $4.2-\mathrm{MeV}$ proton impact where the condition for the applicability of the full peaking approximation $\left(Z_{T} / v \ll<1\right)$ is met. Both theories are very close for $E_{f}>2 \mathrm{keV}$. The fact that near the binaryencounter peak the data for 4.2-MeV $p+\mathrm{He}$ fall so much below theory, is presumably due to experimental problems with the background subtraction at such high electron energies.

Turning to the study of the position of the binaryencounter peak, the experimental values are systematically below the peak position $E_{f}^{*}=2 v^{2} \cos ^{2} \vartheta_{f}+E_{i}$ which results from energy conservation. For the systems from Figs. 2-4, this shift is about $5-10 \%$, increasing slightly with decreasing $v$ or increasing angle. While the peak position predicted by the first-order Born approximation is somewhat lower than $E_{f}^{*}$, but still above experiment, the post as well as the prior IA results are very close to the data (the difference being at most $2 \%$ ). In contrast, the CDW-EIS results [20,21] included in Fig. 3 and also the results from the distorted-wave impulse approximation [8] do not agree so well with experiment.

Figure 5 probes the validity of the post IA for heavier projectiles $\left(5 \mathrm{MeV} / \mathrm{amu} \mathrm{C}^{6+}\right.$ and $\mathrm{O}^{8+}$ ) at nonzero emission angles. A comparison with the experimental data $[15,16]$ shows that in the binary-encounter region and beyond, the post IA works better, the higher the projectile charge, a feature which is also evident from the $0^{\circ}$ spectra of Fig. 2. This indicates that even at emission angles as large as $60^{\circ}$, the emitted high-energy electrons are

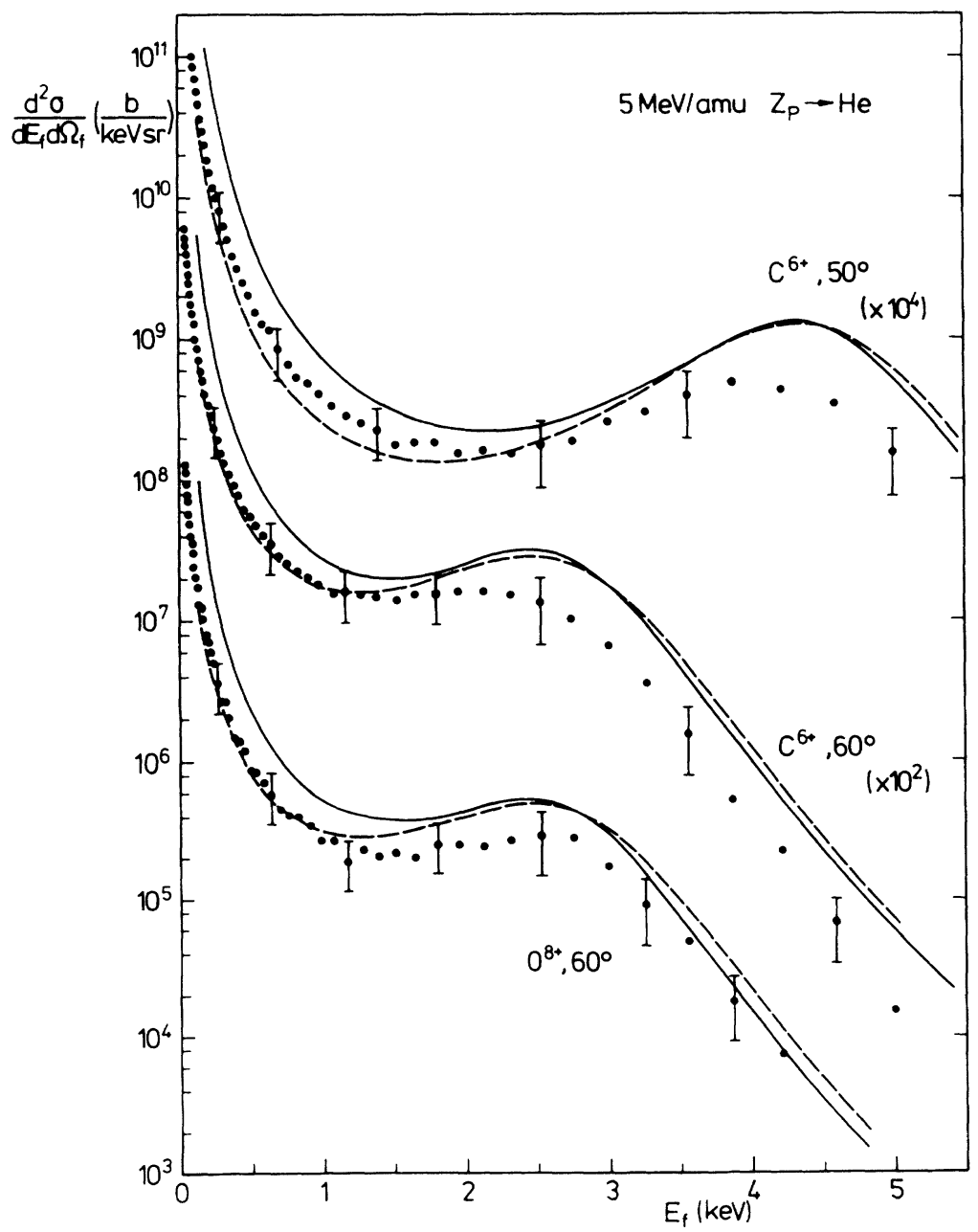

FIG. 5. Doubly differential cross section for electron emission in $5 \mathrm{MeV} / \mathrm{amu}(v=14.15$ a.u.) $\mathrm{C}^{6+}+\mathrm{He}$ at $\vartheta_{f}=50^{\circ}$ and $60^{\circ}$, and $\mathrm{O}^{8+}+\mathrm{He}$ at $\vartheta_{f}=60^{\circ}$. Experiment: Schiwietz et al. [15]. Theory: - transverse peaked post IA, - - - first-order Born. 
largely influenced by the projectile field and hence cannot correctly be described by the first-order Born theory. As far as the position of the binary-encounter peak is concerned, the data do not show the same systematics as those of Figs. 2-4. For the $\mathrm{O}^{8+}+\mathrm{He}$ spectrum, the experimental peak position is, although below $E_{f}^{*}$, above the first-order Born prediction. Moreover, the peak shift relative to $E_{f}^{*}$ is reduced when the projectile charge is increased from 6 to 8 . This behavior is not only at variance with the impulse approximation (which predicts a small increase of the shift with $Z_{P}$ ) but also with other experiments on comparable systems $[5,26]$.

\section{CONCLUSION}

The impulse approximation has been applied to interpret the spectral distribution of the helium electrons emitted in energetic collisions with bare projectiles. Its post version which considers the electron-projectile interaction in the intermediate and in the final state, provides in general a good description of the fast electrons which are emitted into the forward hemisphere, even for hydrogen projectiles. In particular, the region of the binary-encounter peak is very well described except at the highest velocity where experimental uncertainties cannot be excluded. The validity of the transverse peaked post IA has been confirmed in the two regions of its applicability, $Z_{P} / Z_{T} \approx 1$ and $v / Z_{P} \gg 1\left(\mathrm{H}^{+}+\mathrm{He}\right.$ at $v \geq 7.75$ a.u. $)$ as well as $Z_{P} / Z_{T} \gg 1$ and $v \approx Z_{P}\left(\mathrm{O}^{8+}\right.$, $\mathrm{F}^{9+}+\mathrm{He}$ ). It is important, however, to use a HartreeFock-type prescription for the target wave function and potential, and to avoid the full peaking approximation. Results from the DSPB theory are inferior to the present IA results just because of these deficiencies, although the DSPB itself (without approximations) should be considered as a more advanced theory.

Comparison has also been made with the fully peaked prior impulse approximation in the case of light projectiles. For the lower collision velocities, this theory provides a rather good description of the experimental data because it accounts for the final-state interaction of the electron with the target and the projectile field simultaneously. Like the continuum distorted wave theory it is, however, inferior to the post IA at the higher velocities (except for the low-energy electrons).

The ability of the impulse approximation to describe large parts of the spectra of electrons emitted into the forward hemisphere, demonstrates the importance of including nonperturbatively the final-state interaction between electron and projectile in any theoretical description of target ionization, even at high collision energies. This conclusion has also been drawn from a recent coupled-channel investigation [27] of angular-integrated ionization cross sections for proton impact on hydrogen.

\section{ACKNOWLEDGMENTS}

I should like to express my gratitude to J. Macek and K. O. Groeneveld for stimulating discussions, and to C. Fiedler, D. H. Lee, and G. Schiwietz for the communication of unpublished experimental data. I should also like to thank the GSI Darmstadt for supporting this work.
[1] A. Salin, J. Phys. B 2, 631 (1969).

[2] K. Dettmann, K. G. Harrison, and M. W. Lucas, J. Phys. B 7, 269 (1974).

[3] D. R. Bates and G. Griffing, Proc. Phys. Soc. A 66, 961 (1953).

[4] D. H. Lee, P. Richard, T. J. M. Zouros, J. M. Sanders, J. L. Shinpaugh, and H. Hidmi, Phys. Rev. A 41, 4816 (1990).

[5] J. O. P. Pedersen, P. Hvelplund, A. G. Petersen, and P. D. Fainstein, J. Phys. B 23, L597 (1990).

[6] I. M. Cheshire, Proc. Phys. Soc. 84, 89 (1964).

[7] P. D. Fainstein, V. H. Ponce, and R. D. Rivarola, J. Phys. B 21, 287 (1988).

[8] J. E. Miraglia and J. Macek, Phys. Rev. A 43, 5919 (1991).

[9] M. Brauner and J. H. Macek, Phys. Rev. A 46, 2519 (1992).

[10] J. S. Briggs, J. Phys. B 10, 3075 (1977).

[11] D. H. Jakubaßa-Amundsen, J. Phys. B 16, 1767 (1983).

[12] D. H. Jakubaßa-Amundsen, Phys. Rev. A 38, 70 (1988).

[13] K. Taulbjerg, R. O. Barrachina, and J. H. Macek, Phys. Rev. A 41, 207 (1990).

[14] D. H. Lee and P. Richard (private communication).

[15] G. Schiwietz, H. Platten, D. Schneider, T. Schneider, W. Zeitz, K. Musiol, R. Kowallik, and N. Stolterfoht, HMI Berlin Report No. B447, 1987 (unpublished).
[16] H. Platten, G. Schiwietz, T. Schneider, D. Schneider, W. Zeitz, K. Musiol, T. Zouros, R. Kowallik, and N. Stolterfoht, in Abstracts of Contributed Papers, 15th International Conference on the Physics of Electronic and Atomic Collisions, Brighton, 1987, edited by J. Geddes et al. (Queen's University, Belfast, 1987), p. 437.

[17] M. E. Rudd, L. H. Toburen, and N. Stolterfoht, At. Data Nucl. Data Tables 18, 413 (1976).

[18] C. Fiedler, M. Kuzel, and K. O. Groeneveld (private communication).

[19] P. D. Fainstein, V. H. Ponce, and R. D. Rivarola, J. Phys. B 24, 3091 (1991).

[20] P. D. Fainstein, V. H. Ponce, and R. D. Rivarola, J. Phys. B 21, 2989 (1988).

[21] P. D. Fainstein, V. H. Ponce, and R. D. Rivarola, Phys. Rev. A 45, 6417 (1992).

[22] J. N. Madsen and K. Taulbjerg, J. Phys. B (to be published).

[23] J. H. Macek (private communication).

[24] J. Macek and K. Taulbjerg, J. Phys. B 26, 1353 (1993).

[25] J. E. Miraglia and V. H. Ponce, J. Phys. B 13, 1195 (1980).

[26] A. D. Gonzáles, P. Dahl, P. Hvelplund, and P. D. Fainstein, J. Phys. B 26, L135 (1993).

[27] N. Toshima, J. Phys. B 25, L635 (1992). 